\title{
High-resolution proteomic profiling shows sexual dimorphism in zebrafish heart associated proteins
}

\author{
Hamid Niksirat ${ }^{1 *}$, Valentina Siino ${ }^{2}$, Christoph Steinbach ${ }^{1}$, Fredrik Levander ${ }^{2}$ \\ 1 Faculty of Fisheries and Protection of Waters, CENAKVA, University of South Bohemia in \\ České Budějovice, Vodňany, Czech Republic \\ 2 Department of Immunotechnology, Lund University, Lund, Sweden \\ Corresponding author's email address: niksirat@frov.jcu.cz
}

\section{TABLE OF CONTENTS}

The following supporting information is available free of charge at ACS website http://pubs.acs.org

Table S1. Shows peptide and protein identification and quantification in the heart of male and female zebrafish. In peptide tab A column is Peptide Sequence, B column is External IDs accession numbers in UniProt, $\mathrm{C}$ column is peptide Charge, $\mathrm{D}$ column is Average retention time (RT), E column is Average $\mathrm{m} / \mathrm{z}$, $\mathrm{F}$ to $\mathrm{P}$ columns (MH1_01, FH3_01, FH12_01, MH3_01, MH5_01, MH6_01, FH8_01, FH13_01, FH4_01, MH4_01, MH7_01) are intensities of peptides in samples. In protein tab, A column: (V1) is Protein accession numbers, B column: (PepCount) is Peptide count, $C$ column: QPRo is a score for the protein rollup, $D$ column: ( $m-f$ PValue) is male-female $p$-value, $E$ column: ( $m-f$ AdjPValue) is malefemale adjusted $p$-value (q-value), $F$ column: ( $m$-f_log2FoldChange) is male-female log2 Fold Change (negative and positive values are female and male biased, respectively), G column: (featureAvg) is feature Average, $\mathrm{H}$ to R columns (MH1_01, FH3_01, FH12_01, MH3_01, MH5_01, MH6_01, FH8_01, FH13_01, FH4_01, MH4_01, MH7_01) are log2-transformed and normalized protein level intensities for the different samples. The name of each sample can be seen in Design tab of the Table S1. S column: gene name, $\mathrm{T}$ column: protein name.

Table S2. Detailed functions of proteins significantly $(q<0.05)$ different in the heart of male and female zebrafish. Uncharacterized proteins were annotated using BLAST in UniProt to determine their potential functions.

Table. S3. A comparative table that shows similar trends of sexual dimorphism in zebrafish plasma protein abundances $(p<0.05)$ which were obtained from Li et al., 2016 and present study. $F=$ Female, $\mathrm{M}=$ Male.

Table S4. List of proteins enriched in biological process and molecular function pathways using significantly different proteins between male and female zebrafish heart created by ShinyGO (v0.61).

Table S5. Detailed information of network map shows interaction among query nodes (differentially expressed proteins passing the q-value cut-off 0.05) created using STRING (v11.0). Interactions are displayed for experimentally determined, curated database, text mining, gene neighborhood, gene co-occurrence, and co-expression. 
Figure S1. High-resolution STRING network map shows interaction among query nodes. Network is a summary view shows current interactions containing following items: Purple line - experimental evidence: co-purification, co-crystallization, Yeast 2-Hybrid, genetic interactions, etc. as imported from primary sources. Light blue line - database evidence: known metabolic pathways, protein complexes, signal transduction pathways, etc. from curated databases. Yellow line - textmining evidence: Automated textmining, searching for proteins that are frequently mentioned together in literature. Green line - neighbourhood evidence: groups of genes that are frequently observed in each other's genomic neighbourhood. Blue line - cooccurrence evidence: gene families whose occurrence patterns across genomes show similarities. Black line - coexpression evidence: proteins whose genes are observed to be correlated in expression, across a large number of experiments. 
Table S2. Detailed functions of proteins significantly $(q<0.05)$ different in the heart of male and female zebrafish. Uncharacterized proteins were annotated using BLAST in UniProt to determine their potential functions.

\begin{tabular}{|c|c|c|}
\hline $\begin{array}{l}\text { Accession } \\
\text { number }\end{array}$ & Protein & Function \\
\hline E7F2M5 & $\begin{array}{l}\text { CD59 molecule (CD59 blood } \\
\text { group) }\end{array}$ & Regulator of immune (complement) system \\
\hline F1QV15 & Vitellogenin 6 & Lipid transport \\
\hline A0A0R4IPQ8 & Complement factor $\mathrm{H}$ & Regulation of complement immune response \\
\hline F1Q7L0 & Vitellogenin 4 & Lipid transport \\
\hline F1REF9 & Proteoglycan $4 \mathrm{~b}$ & Immune response, scavenger receptor \\
\hline Q1LWN2 & Vitellogenin 1 & Lipid transport \\
\hline X1WGQ3 & Si:dkey-96g2.1 & $\begin{array}{l}\text { A0A498LYM0 (35.7\%): Paired box Pax-3 isoform } \\
\text { X3 (rohu_029833), sequence-specific DNA } \\
\text { binding, multicellular organism development }\end{array}$ \\
\hline Q1MTC4 & Vitellogenin 2 & Lipid transport \\
\hline 042364 & Apolipoprotein Eb & Lipid transport \\
\hline X1WE71 & Transferrin-a & Iron transport \\
\hline F1Q890 & Plasminogen & Blood coagulation \\
\hline F6NTZ9 & Alpha-2-macroglobulin-like & $\begin{array}{l}\text { Endopeptidase inhibitor, blood coagulation, } \\
\text { regulation of complement activation and } \\
\text { immune response }\end{array}$ \\
\hline AOA0R4IU44 & $\begin{array}{l}\text { Inter-alpha-trypsin inhibitor } \\
\text { heavy chain } 3 b\end{array}$ & $\begin{array}{l}\text { Endopeptidase inhibitor, reduction of } \\
\text { extracellular histone toxicity }\end{array}$ \\
\hline BOUXNO & Sulfhydryl oxidase & $\begin{array}{l}\text { Antioxidant, protein folding and calcium } \\
\text { handling during stress }\end{array}$ \\
\hline B8JL43 & Serotransferrin & Iron transport \\
\hline F1R8N2 & Sb:cb37 & Endopeptidase inhibitor, \\
\hline F1QLC3 & Coagulation factor $\mathrm{X}$ & Blood coagulation \\
\hline F1R2S5 & Vitellogenin 5 & Lipid transport \\
\hline F1RAP6 & $\begin{array}{l}\text { Serpin peptidase inhibitor, } \\
\text { clade } \mathrm{F} \text { (alpha- } 2 \text { antiplasmin, } \\
\text { pigment epithelium-derived } \\
\text { factor), member } 2 \mathrm{~b}\end{array}$ & Blood coagulation \\
\hline X1WC44 & Si:ch211-212c13.8 & $\begin{array}{l}\text { Endopeptidase inhibitor, Platelet degranulation, } \\
\text { clot formation, extracellular matrix degradation, } \\
\text { Rho GTPase cycle, HDL assembly }\end{array}$ \\
\hline E7FAN5 & $\begin{array}{l}\text { Coagulation factor II } \\
\text { (thrombin) }\end{array}$ & Blood coagulation \\
\hline F1R442 & $\begin{array}{l}\text { Arachidonate } 5 \text { - } \\
\text { lipoxygenase } b \text {, tandem } \\
\text { duplicate } 3\end{array}$ & Immune system product against oxidized LDL \\
\hline Q8JH29 & Angiotensinogen & Blood pressure \\
\hline
\end{tabular}




\begin{tabular}{|c|c|c|}
\hline Q6DEI0 & $\begin{array}{l}\text { Phospholipid transfer } \\
\text { protein }\end{array}$ & Phospholipid transport \\
\hline A5PMP6 & $\begin{array}{l}\text { Complement component } \\
\text { c3b, tandem duplicate } 1\end{array}$ & Immune system \\
\hline B0S5N7 & Biliverdin reductase $\mathrm{A}$ & Antioxidant \\
\hline B0S642 & $\begin{array}{l}\text { 3-hydroxyisobutyryl-CoA } \\
\text { hydrolase, mitochondrial }\end{array}$ & Catabolic process of branched-chain amino acids \\
\hline AOAOR4ISS9 & $\begin{array}{l}\text { Protein C (inactivator of } \\
\text { coagulation factors Va and } \\
\text { VIIIa), a }\end{array}$ & Blood coagulation \\
\hline F8W4E2 & $\begin{array}{l}\text { Sphingomyelin } \\
\text { phosphodiesterase, acid-like } \\
\text { 3B }\end{array}$ & Immune system, inflammation \\
\hline Q6DGJ3 & $\begin{array}{l}\text { Mitochondrial import inner } \\
\text { membrane translocase } \\
\text { subunit Tim13 }\end{array}$ & $\begin{array}{l}\text { Chaperone-mediated protein transport, protein } \\
\text { insertion into mitochondrial inner membrane, } \\
\text { regulation of stress }\end{array}$ \\
\hline Q7ZUP0 & $\begin{array}{l}\text { Acidic leucine-rich nuclear } \\
\text { phosphoprotein } 32 \text { family } \\
\text { member A }\end{array}$ & $\begin{array}{l}\text { Histone binding, cell cycle and survival, } \\
\text { regulation of apoptosis, vascular development, } \\
\text { immune response }\end{array}$ \\
\hline Q90486 & Hemoglobin subunit beta-1 & Oxygen transport \\
\hline Q9PT95 & Retinol binding protein & Retinol (vitamin A) carrier \\
\hline A0A0R4IS53 & $\begin{array}{l}\text { GTPase-activating protein } \\
\text { and VPS9 domains } 1\end{array}$ & Phagocytosis \\
\hline F1QV29 & $\begin{array}{l}\text { Complement component } \\
\text { c3a, duplicate } 2\end{array}$ & Immune system \\
\hline A0JMK3 & Zgc:153913 & $\begin{array}{l}\text { P22792(31.2\%): Carboxypeptidase N subunit } 2 \\
\text { (cpn2), Regulation of complement activation, } \\
\text { Protein stabilization, Enzyme regulation. } \\
\text { Q80X72 (29.9\%): Leucine-rich repeat-containing } \\
\text { protein } 15 \text { (Irrc15), Protein localization to plasma } \\
\text { membrane, Cell migration, Virion attachment to } \\
\text { host cell, Collagen, fibronectin and laminin } \\
\text { binding. } \\
\text { O08742 (28\%): Platelet glycoprotein V (gp5), } \\
\text { Blood coagulation, Cell-matrix adhesion, Platelet } \\
\text { activation. }\end{array}$ \\
\hline F6NYT7 & Glutathione peroxidase & Antioxidant \\
\hline Q800W7 & $\begin{array}{l}\text { Uroporphyrinogen III } \\
\text { synthase }\end{array}$ & Heme biosynthesis \\
\hline
\end{tabular}




\begin{tabular}{|c|c|c|}
\hline F1Q5S6 & $\begin{array}{l}\text { AarF domain-containing } \\
\text { kinase } 5\end{array}$ & Mitochondria fission \\
\hline A0A2R8Q0U7 & $\begin{array}{l}\text { Choline transporter-like } \\
\text { protein } 2\end{array}$ & $\begin{array}{l}\text { Choline transport, antioxidant, prevent } \\
\text { autoimmune disorders, hemostasis }\end{array}$ \\
\hline F1R945 & Si:ch211-197g15.10 & Immune response, GTP binding \\
\hline Q7ZVG7 & Fgg protein & Blood coagulation \\
\hline Q5RHE5 & Si:dkey-90m5.4 & $\begin{array}{l}\text { P02750 (31.5\%\%) Leucine-rich alpha-2- } \\
\text { glycoprotein (Irg1), brown fat cell differentiation, } \\
\text { neutrophil degranulation, Angiogenesis, } \\
\text { endothelial cell proliferation, transforming } \\
\text { growth factor beta receptor signaling pathway. }\end{array}$ \\
\hline B8A5L6 & Fibrinogen alpha chain & Blood coagulation \\
\hline Q6DGJ6 & Peroxiredoxin 2 & Antioxidant \\
\hline B3DIT9 & $\begin{array}{l}\text { Pyruvate dehydrogenase } \\
\text { kinase, isozyme } 4\end{array}$ & Glycolysis pathway \\
\hline Q6P5L3 & 60S ribosomal protein L19 & $\begin{array}{l}\text { Protein expression, unfolded protein response } \\
\text { against apoptosis }\end{array}$ \\
\hline Q6PFT2 & C6 protein & Immune (complement) system \\
\hline Q6ZM17 & $\begin{array}{l}\text { Novel protein similar to } \\
\text { zebrafish hemoglobin alpha- } \\
\text { adult } 1 \text { (Hbaa1) }\end{array}$ & Oxygen transport \\
\hline A0A0R4IQ55 & $\begin{array}{l}\text { Hydroxymethylbilane } \\
\text { synthase a }\end{array}$ & Heme biosynthetic process \\
\hline Q92051 & Carbonic anhydrase & Oxygen transport \\
\hline Q5MPX4 & Transferrin receptor $1 \mathrm{a}$ & Iron transport \\
\hline A8KBJ5 & Histone H3 & $\begin{array}{l}\text { Nucleosome assembly, neutrophil extracellular } \\
\text { trap }\end{array}$ \\
\hline B8JMC1 & Biliverdin reductase $B$ & Antioxidant \\
\hline E9QEK2 & Tpd52-like 2b & Protein homodimerization \\
\hline Q92005 & Elongation factor 1-alpha & $\begin{array}{l}\text { Protein synthesis, nuclear export process, } \\
\text { proteolysis, cytoskeleton organization, } \\
\text { apoptosis, antioxidant }\end{array}$ \\
\hline E7FC32 & Tubulin beta chain & Cytoskeleton \\
\hline F1QAA3 & Zgc:153659 & Immune response, Immunoglobulin production \\
\hline
\end{tabular}




\begin{tabular}{|c|c|c|}
\hline F1R2W1 & $\begin{array}{l}\text { Dehydrogenase/reductase } \\
\text { (SDR family) member 13a, } \\
\text { duplicate } 3\end{array}$ & Antioxidant \\
\hline F6NT71 & $\begin{array}{l}\text { Hemoglobin beta } \\
\text { embryonic-1.1 }\end{array}$ & Oxygen transport \\
\hline Q90487 & Hemoglobin subunit alpha & Oxygen transport \\
\hline AOA0R4IY13 & Si:ch1073-416d2.3 & Serine-type endopeptidase inhibitor \\
\hline F1QYT2 & $\begin{array}{l}\text { Sulfide quinone } \\
\text { oxidoreductase }\end{array}$ & $\mathrm{H} 2 \mathrm{~S}$ detoxification \\
\hline A0A2R8QLD1 & $\begin{array}{l}\text { Phosphorylase } b \text { kinase } \\
\text { regulatory subunit }\end{array}$ & Glycogen metabolism \\
\hline A0A2R8Q476 & $\begin{array}{l}\text { ATP-dependent 6- } \\
\text { phosphofructokinase }\end{array}$ & Glycolysis pathway \\
\hline F1Q4N7 & $\begin{array}{l}\text { Acidic (leucine-rich) nuclear } \\
\text { phosphoprotein } 32 \text { family, } \\
\text { member B }\end{array}$ & $\begin{array}{l}\text { Histone binding, cell cycle and survival, } \\
\text { regulation of apoptosis, vascular development, } \\
\text { immune response }\end{array}$ \\
\hline A0A0R4IQ34 & Si:dkey-93h22.7 & $\begin{array}{l}\text { Q96RD9 (24.1\%): Fc receptor-like protein } 5 \\
\text { (fcrl5), Immune response. } \\
\text { P51866 (22.2\%): Platelet endothelial cell } \\
\text { adhesion molecule (pecam1), Cell adhesion for } \\
\text { immune cell transendothelial migration, } \\
\text { phosphorylation, phagocytosis. }\end{array}$ \\
\hline F1QYR3 & Uncharacterized protein & Unknown \\
\hline B8JIS8 & $\begin{array}{l}\text { Glutathione S-transferase } \\
\text { mu, tandem duplicate } 1\end{array}$ & Antioxidant \\
\hline Q6NYE1 & Fibrinogen beta chain & Blood coagulation \\
\hline BOROK9 & Si:ch211-103n10.5 & $\begin{array}{l}\text { Nucleosome assembly, neutrophil extracellular } \\
\text { trap }\end{array}$ \\
\hline Q1L8D8 & Si:rp71-15k1.1 & $\begin{array}{l}\text { Q58714 (25.1\%): Uncharacterized protein } \\
\text { MJ1318 (mj1318), ATP binding, Serine-type } \\
\text { endopeptidase activity, Protein quality control. }\end{array}$ \\
\hline Q6YLH9 & Caveolin & Intracellular transport, Calcium signaling \\
\hline BOV2R7 & $\begin{array}{l}\text { Serpin peptidase inhibitor, } \\
\text { clade } \mathrm{G} \text { ( } \mathrm{C} 1 \text { inhibitor), } \\
\text { member } 1\end{array}$ & Blood coagulation \\
\hline F1QFC2 & Complement factor B & Immune system \\
\hline A0A2R8Q886 & Glypican 1b & $\begin{array}{l}\text { Cell migration and growth, } \\
\text { Cell surface }\end{array}$ \\
\hline
\end{tabular}




\begin{tabular}{|l|l|l|}
\hline A0A0R4ITA8 & $\begin{array}{l}\text { ATP-dependent 6- } \\
\text { phosphofructokinase }\end{array}$ & Glycolysis pathway \\
\hline B8JL30 & High mobility group box 2a & DNA repair, heart regeneration, inflammation \\
\hline F1Q5C6 & Exportin 7 & Protein export from nucleus \\
\hline
\end{tabular}


Table. S3. A comparative table that shows similar trends of sexual dimorphism in zebrafish plasma protein abundances $(p<0.05)$ which were obtained from Li et al., 2016 and present study. $F=$ Female, $M=$ Male.

\begin{tabular}{|c|c|c|}
\hline Protein & Trend & Function \\
\hline Vitellogenin 1 & $\mathrm{~F}>\mathrm{M}$ & Lipid transport \\
\hline Vitellogenin 2 & $\mathrm{~F}>\mathrm{M}$ & Lipid transport \\
\hline Vitellogenin 4 & $\mathrm{~F}>\mathrm{M}$ & Lipid transport \\
\hline Vitellogenin 5 & $\mathrm{~F}>\mathrm{M}$ & Lipid transport \\
\hline Vitellogenin 6 & $\mathrm{~F}>\mathrm{M}$ & Lipid transport \\
\hline Apolipoprotein Eb & $\mathrm{F}>\mathrm{M}$ & Lipid transport \\
\hline Apolipoprotein A-IV & $\mathrm{F}>\mathrm{M}$ & Lipid transport \\
\hline Coagulation factor & $\mathrm{F}>\mathrm{M}$ & Blood coagulation \\
\hline Retinol binding protein 4 & $\mathrm{~F}>\mathrm{M}$ & Retinol (vitamin A) carrier \\
\hline Complement C4-B & $M>F$ & Immune system \\
\hline Alpha-2-macroglobulin-like & $\mathrm{F}>\mathrm{M}$ & $\begin{array}{c}\text { Endopeptidase inhibitor, blood } \\
\text { coagulation, regulation of } \\
\text { complement activation and } \\
\text { immune response }\end{array}$ \\
\hline Hemoglobin subunit beta-1 & $\mathrm{F}>\mathrm{M}$ & Oxygen transport \\
\hline Sulfhydryl oxidase & $\mathrm{F}>\mathrm{M}$ & $\begin{array}{l}\text { Antioxidant, protein folding } \\
\text { and calcium handling during } \\
\text { stress }\end{array}$ \\
\hline Serotransferrin & $\mathrm{F}>\mathrm{M}$ & Iron transport \\
\hline Hemopexin & $M>F$ & Heme binding \\
\hline $\begin{array}{l}\text { Inter-alpha-trypsin inhibitor } \\
\text { heavy chain }\end{array}$ & $\mathrm{F}>\mathrm{M}$ & $\begin{array}{c}\text { Endopeptidase inhibitor, } \\
\text { reduction of extracellular } \\
\text { histone toxicity }\end{array}$ \\
\hline Fetuin B & $\mathrm{F}>\mathrm{M}$ & Protease inhibitor \\
\hline $\begin{array}{c}\text { Complement component c3a, } \\
\text { duplicate } 2\end{array}$ & $\mathrm{~F}>\mathrm{M}$ & Immune system \\
\hline Alpha-2-antiplasmin & $\mathrm{F}>\mathrm{M}$ & Blood coagulation \\
\hline
\end{tabular}




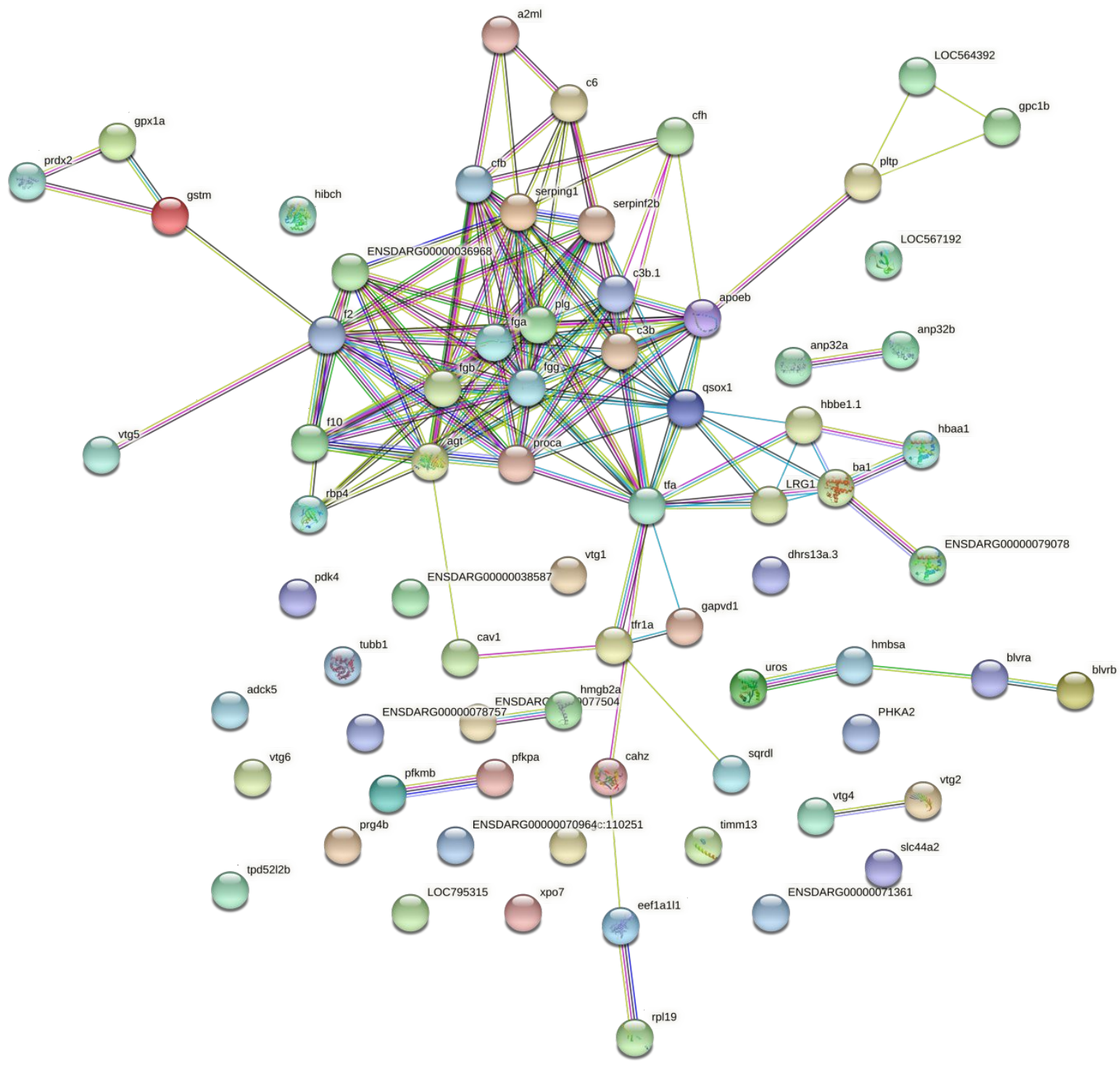

Figure S1. High-resolution STRING network map shows interaction among query nodes. Network is a summary view shows current interactions containing following items: Purple line - experimental evidence: co-purification, co-crystallization, Yeast 2-Hybrid, genetic interactions, etc. as imported from primary sources. Light blue line database evidence: known metabolic pathways, protein complexes, signal transduction pathways, etc. from curated databases. Yellow line - textmining evidence: Automated textmining, searching for proteins that are frequently mentioned together in literature. Green line - neighbourhood evidence: groups of genes that are frequently observed in each other's genomic neighbourhood. Blue line - cooccurrence evidence: gene families whose occurrence patterns across genomes show similarities. Black line - coexpression evidence: proteins whose genes are observed to be correlated in expression, across a large number of experiments. 